\title{
The Effects of Corneal Edema on the Intraocular
}

\section{Pressure Measurements}

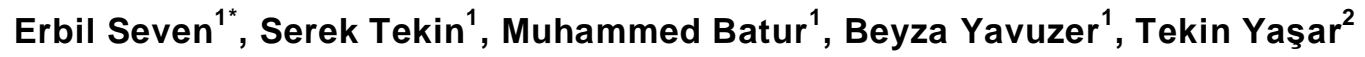 \\ ${ }^{1}$ Department of Ophthalmology, V an Yüzüncü Yul University, Van, Turkey \\ ${ }^{2}$ Health Sciences University, Beyoglu Eye Training and Research Hospital, İstanbul, Turkey
}

\begin{abstract}
We aimed to evaluate the possible effects of corneal edema on intraocular pressure (IOP) measurements obtained with three different tonometers on the first day after cataract surgery.

Sixty-four eyes of the 64 patients underwent phacoemulsification and IOP measurements using Goldmann applanation tonometer (GAT), Tono-Pen applanation tonometer (TAT), and Pascal digital contour tonometer (DCT) the first day after surgery. Central corneal thicknes s (CCT) measurements were used to quantify the postoperative corneal edema. Intraclass correlation coefficient and Bland-Altman plot were used to assess the compatibility of IOP measurements among three method pairs.

TAT and DCT IOP measurements were found to be compatible with each other $(p=0.141)$ in the corneal edema group. The GAT and TAT IOP measurements in the eyes with corneal edema were inversely proportional to the CCT values $(p=0.011$ and $p=0.033$, respectively), but the DCT relationship was not statistically significant $(\mathrm{p}=0.44)$.

The lack of a significant relationship between DCT and CCT in edematous corneas may suggest that DCT, as in normal corneas, can perform CCT independent measurements. However, there is still no gold standard method for IOP measurement in edematous corneas.
\end{abstract}

Key Words: corneal edema, digital contour tonometer, Goldmann applanation tonometer, intraocular pressure, Tono-Pen

\section{Introduction}

The intraocular pressure (IOP) is one of the most significant measurement parameters in an ophthalmological examination, especially during a glaucoma follow-up. Goldmann applanation tonometry (GAT) is still used as the gold standard for measuring the IOP $(1,2)$. However, GAT does have some limitations, and its measurements can be affected by the central corneal thickness (CCT), refractive surgery, topical anesthetics, fluorescein dye, corneal surface irregularities, and tear film (3). Previous researches have shown that there is a positive correlation between GAT IOP measurements and the CCT (4). For example, Schneider and Grehn found a linear correlation, with an increase in the GAT results of $4.5 \mathrm{mmHg}$ per $100 \mu \mathrm{m}$ of the CCT (5). Kohlhaas et al. suggested that the IOP readings should be adjusted by the corneal thickness according to the Dresden correction table in order to obtain the true IOP values (6). The Dresden correction formula defines an approximately $0.4 \mathrm{mmHg}$ adjustment per $10 \mu \mathrm{m}$ of deviation from a CCT of $550 \mu \mathrm{m}$ (6).

The Tono-Pen (Medtronic Surgical Technologies, Jacksonville, Florida, USA) is a portable tonometer that uses a solid strain gauge transducer (7). Based on the literature, Tono-Pen applanation tonometry (TAT) IOP measurements have been affected by the
CCT, and there was a positive correlation between them $(8,9)$. However, Bhan et al. found that the CCT impact on the IOP measurements was less when using TAT than when using GAT (10).

The Pascal dynamic contour tonometer (SMT Swiss Microtechnology AG, Port, Switzerland) is a nonapplanation, slit-lamp-mounted, digital contact tonometer. It has been reported that this device can measure the IOP irrespective of the corneal characteristics (11).

It has been shown that the corneal biomechanical properties can affect the IOP measurements (12). Corneal edema, in particular, can cause different IOP measurements $(13,14)$. Therefore, the aim of this study was to compare the IOP values obtained by GAT, TAT, and Pascal dynamic contour tonometer in patients with postoperative corneal edema associated with cataract surgery in order to determine whether these measurements are related with the CCT.

\section{Materials and Methods}

This prospective, observational study was conducted in Van Yüzüncü Yll University ophthalmology clinic. All of the procedures performed in this study involving human participants were in accordance with the ethical standards of the Declaration of Helsinki, 


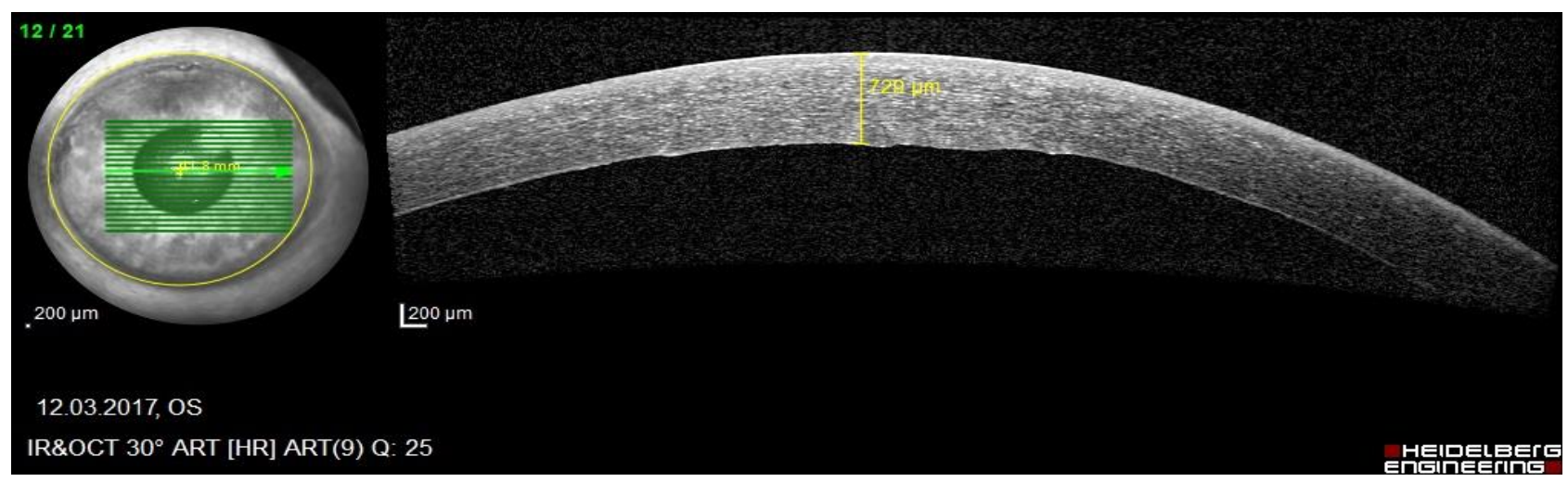

Fig. 1. Measurement of central corneal thickness with anterior segment optical coherence tomography

and this study protocol was approved by the Ethics Committee of Yüzüncü Yil University in Van, Turkey. Seventy patients with advanced cataracts who underwent phacoemulsification surgery were included in this study. Six of the patients were subsequently excluded because they were noncompliant with the IOP measurements or the quality of the measurements was not high enough when using Pascal dynamic contour tonometry (DCT) or TAT. Those patients with glaucoma before surgery, bullous keratopathy, corneal opacity or scar, corneal ectasia, positive Seidel test, contact lens wear, corneal astigmatism $>3$ diopters, and any intraoperative complications were excluded from this study. All of the eyes with stromal edema were included in the study. The patients' fellow eyes made up the control group. The same exclusion criteria applied to the control group. Edematous eyes defined as group 1, and non-edematous fellow eyes (control group) as group 2.

Surgical Procedure: Each of the study subjects was applied local (sub-Tenon) anesthesia before surgery. Superotemporal (for right eyes) or superonasal (for left eyes) clear cornea incision constructed with a 2.2 $\mathrm{mm}$ cornea knife. The flap was created using a cystotome and continuous circular capsulorhexis completed with the capsule forceps. Two side ports were created with a 20 -gauge micro-vitreoretinal knife in the horizontal axis (temporal-nasal). Hydrodissection in different directions, divide and conquer technique used to deal with a central nucleus. Cortex removal was performed with bimanual irrigation and aspiration before intracapsular implantation of a foldable acrylic intraocular lens (AcrySof SA60AT; Alcon Laboratories, Inc., Fort Worth, TX, USA). Intracameral cefuroxime was applied and corneal incisions were hydrated. A combination of cohesive and dispersive ophthalmic viscosurgical device (4\% sodium chondroitin sulfate, $1.65 \%$ sodium hyaluronate) was utilized before the capsulorhexis and the intraocular lens implantation stages. None of the patients required the corneal suture. All of the operations were carried out by experienced surgeons (ES, MB, and ST), and the phacoemulsification was followed by the intracapsular implantation in all of the subjects.

All of the examinations were carried out by one physician (BY) in the same order on the first day after surgery. After a detailed examination of the anterior segment of the eye was conducted using a slit-lamp biomicroscope. Fine slit beam illumination angled at $45^{\circ}$ was used for a detailed view of the corneal layers and corneal edema. The corneal edema was diagnosed clinically. Corneal edema was described as the loss of transparency in the thickened cornea with or without Descemet folds. CCT was measured followed by the IOP readings (GAT, TAT, and DCT, in that order). The IOP readings were conducted once using each method. Then, the fellow eye measurements were obtained, in the same order. No correction was performed in IOP readings obtained tonometers in edematous eyes and fellow non-edematous eyes for statistical analysis.

The CCT was determined using anterior segment optical coherence tomography (AS-OCT) (Spectralis; Heidelberg Engineering $\mathrm{GmbH}$, Heidelberg, Germany) by one examiner (ES) (Figure 1). The IOP was measured one day after the cataract surgery using GAT (AT 900; Haag-Streit AG, Köniz, Switzerland), TAT, and DCT. The GAT and DCT devices were mounted on the slit-lamp biomicroscope. The IOP readings were taken while the patient was in an upright sitting position after the cornea was anesthetized with $0.5 \%$ proparacaine hydrochloride eye drops (Alcaine; Alcon Pharmaceuticals Ltd., Fribourg, Switzerland). Five minutes break was taken between the usage of the different devices and between the repeated measurements with the same tonometer. Fluorescein drops were instilled into the eye before GAT measurements. The GAT readings were always taken first in order to prevent the operator bias associated with the TAT and DCT measurements. Three readings were taken, and the 

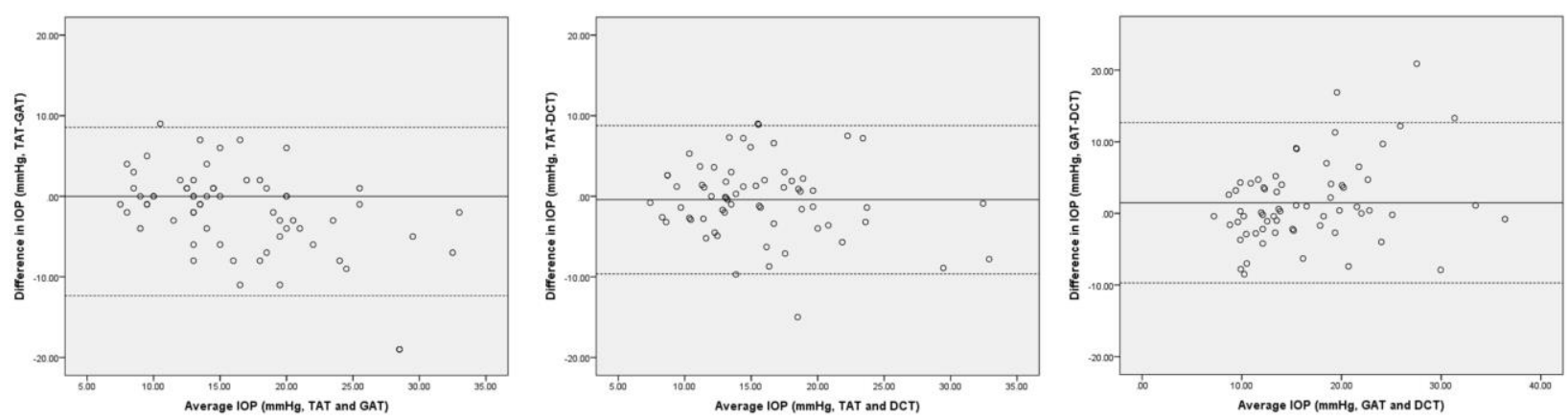

Figure 2a
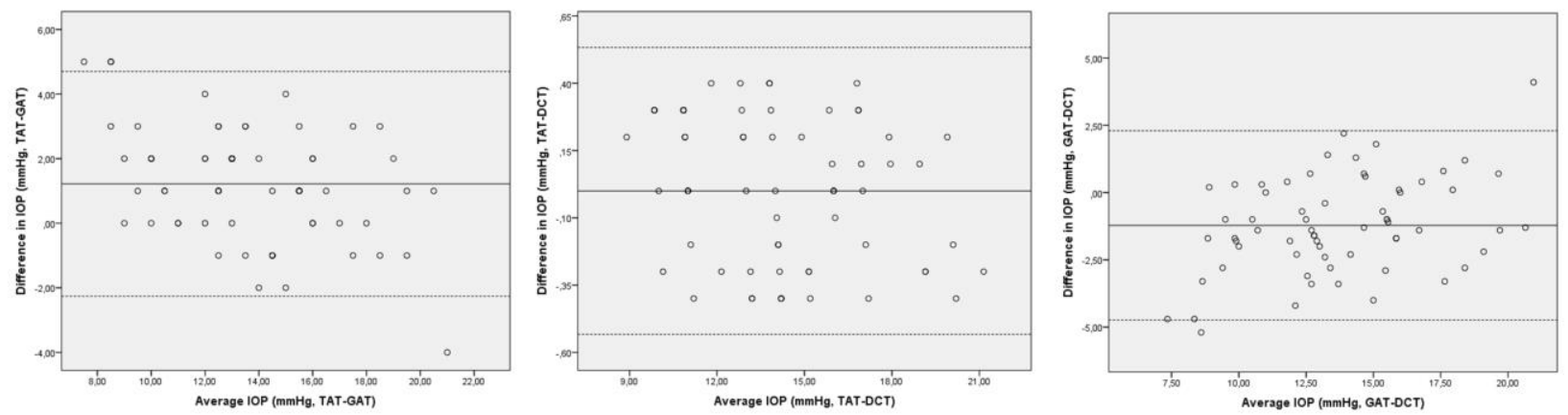

Figure 2b

Fig. 2a. Bland-Altman plot showing the difference against the mean of IOP measurement method pairs and standard measurements with limits of agreement (broken lines) separately in Group 1 (Edematous eyes)

Fig. 2b. Bland-Altman plot showing the difference against the mean of IOP measurement method pairs and standard measurements with limits of agreement (broken lines) separately in Group 2 (Nonedematous fellow eyes/ control group)

average of these readings was computed. The TonoPen takes five independent readings in order to provide a reliability index for each measurement, and the readings were considered valid only if the scatter index was $<5 \%$. Three valid readings were accepted, and the average of these readings was computed. The DCT readings consisted of at least five oscillations, and the readings were accepted only if the quality index score was three or better.

Statistical Analysis: In this study, IBM SPSS Statistics for Windows version 23.0 (IBM Corp., Armonk, NY, USA) was used for the data analysis. The Kolmogorov-Smirnov test was used to determine whether the data conformed to a normal distribution. A basic linear regression was used to test for variations in the IOP measurements according to the CCT in both groups using all three devices. Intraclass correlation coefficient and Bland-Altman plot were used to assess the compatibility of IOP measurements among three method pairs. The paired samples t-test was used for the between-group comparisons (the postoperative edematous eyes were in Group 1 and the non-edematous fellow eyes were in Group 2) according to the CCT, GAT IOP, TAT IOP, and DCT IOP measurements. A p-value $<0.05$ was

\section{Results}

In this study, 128 eyes (64 eyes with edema and 64 normal fellow eyes without edema) of 64 patients were enrolled. The mean age of the patients was $70.53 \pm 10.12$ years old (range: $37-90$ years). Thirty-six of the patients were males $(56.3 \%)$ and 28 were females $(43.8 \%)$. Comparison of CCT and IOP measurements in postoperative edematous eyes (Group 1) and control eyes (Group 2) are shown in Table 1.

IOP measurements obtained using TAT and DCT were found as compatible with each other in Group 1 $(\mathrm{p}=0.141)$. IOP measurements obtained using TAT and GAT, GAT and DCT were not found as compatible with each other in Group $1(p<0.001$, $\mathrm{p}=0.013$ respectively). IOP measurements obtained using TAT and DCT were found as compatible with each other in Group 2 ( $p=0.053)$. A comparison of the compatibility of the three method pairs in both groups is shown in Table 2. Bland-Altman plot showing the difference against the mean of IOP measurement method pairs and standard 

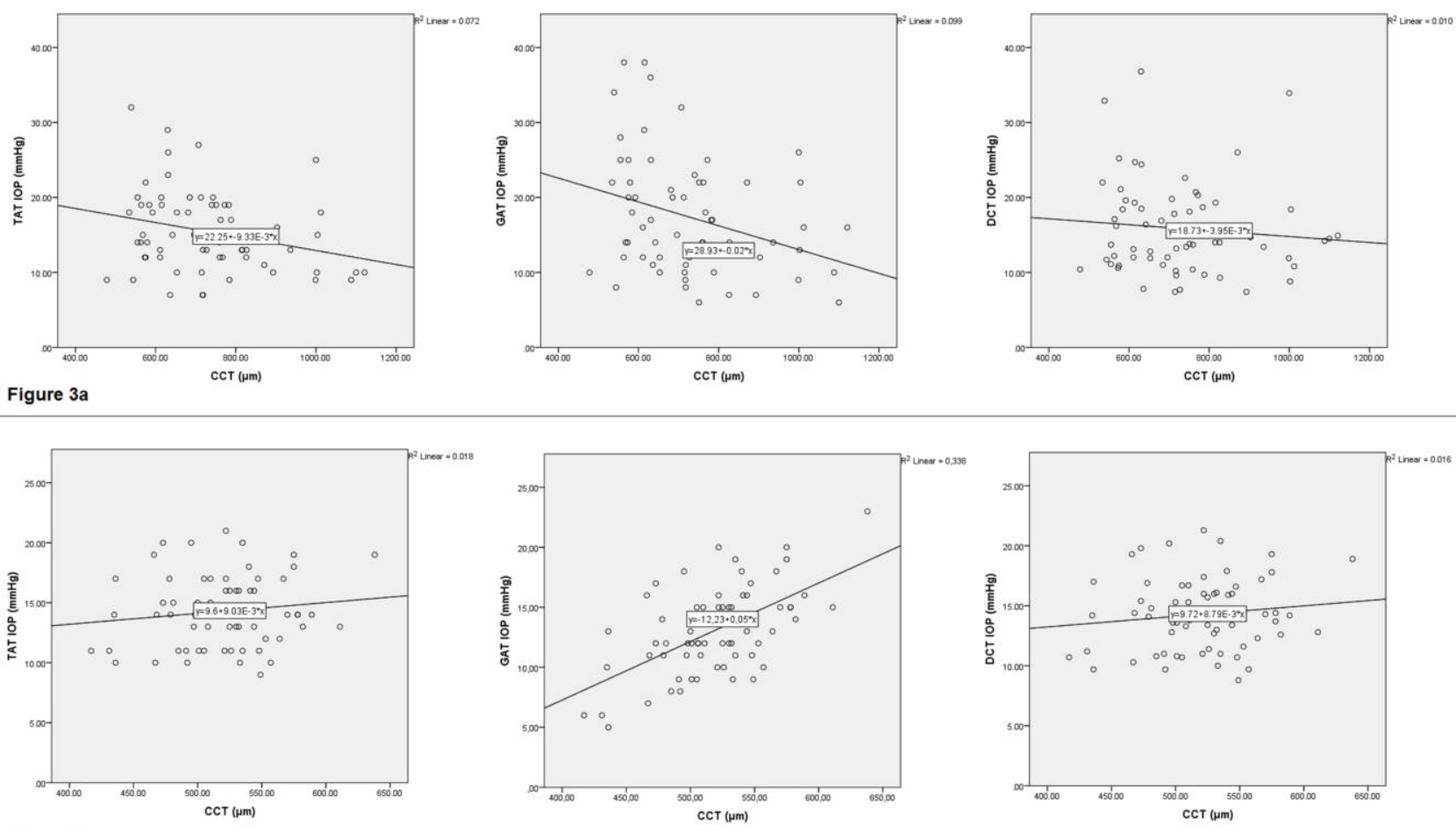

Figure 3b

Fig. 3a. Intraocular pressure measurements made with Goldmann applanation tonometer, Tono-Pen, and Digital contour tonometer plotted against central corneal thickness in Group 1 (Edematous eyes)

Fig. 3b. Intraocular pressure measurements made with Goldmann applanation tonometer, Tono-Pen, and Digital contour tonometer plotted against central corneal thickness in Group 2 (Nonedematous fellow eyes/ control group)

measurements with limits of agreement (broken lines) separately in Figure 2.

The changes in the IOP per $100 \mu \mathrm{m}$ change in the CCT were $-1.6 \mathrm{mmHg}(\mathrm{p}=0.011),-0.9 \mathrm{mmHg}(\mathrm{p}=$ 0.033), and $-0.4 \mathrm{mmHg}(\mathrm{p}=0.44)$ for the GAT, TAT, and DCT, respectively, in Group 1. In Group 2, the changes in the IOP per $100 \mu \mathrm{m}$ change in the CCT were $+4.9 \mathrm{mmHg}(\mathrm{p}<0.001),+0.9 \mathrm{mmHg}(\mathrm{p}=$ $0.29)$, and $+0.9 \mathrm{mmHg}(\mathrm{p}=0.32)$ for the GAT, TAT, and DCT, respectively. The interquartile ranges for the IOP were $10 \mathrm{mmHg}, 7 \mathrm{mmHg}$, and $7.9 \mathrm{mmHg}$ for the GAT, TAT, and DCT, respectively, in Group 1. In Group 2, the interquartile ranges for the IOP were $4.00 \mathrm{mmHg}, 5.5 \mathrm{mmHg}$, and $5.03 \mathrm{mmHg}$ for the GAT, TAT, and DCT, respectively. There were inverse correlations between the GAT IOP and CCT in Group $1(\mathrm{p}=0.011)$ and a correlation Group 2 $(p<0.001)$. There was also an inverse correlation between the TAT IOP and CCT in Group 1 ( $\mathrm{p}=$ 0.033).

The variations in the IOP measurements for all three devices according to the CCT for both groups are shown in Figure 3.

\section{Discussion}

In our study, there was significant accordance between TAT and DCT measurements in Group 1 ( $p$
$=0.141)$. The mean IOP values obtained using the TAT and GAT, GAT and DCT were not significantly compatible with each other in Group $1(\mathrm{p}<0.001$, $\mathrm{p}=0.013$ respectively). Goldmann applanation tonometry (GAT) is accepted as the "gold standard" method for IOP evaluation (2). In the present study, the fact that DCT and TAT were more compatible does not mean that their measurements were more accurate because corneal edema causes a change in the biomechanical properties of the cornea. Therefore, the gold standard method in eyes with corneal edema is still unclear. Studies comparing intraocular manometric measurements with other tonometry measurements may be more informative. IOP measurements obtained using TAT and DCT was found as compatible with each other in Group 2 $(\mathrm{p}=0.053)$. This accordance was only between DCT and TAT in normal eyes. That might be due to the fact that no correction table is used in GAT measurements according to CCT. The variations among IOP measurements obtained using different tonometers in edematous eyes were also higher than normal eyes (Intracorrelation coefficients interval: $0.80-0.81$ in edematous eyes, $0.89-1.00$ in normal eyes).

Another result of our study was seen in the linear regression analysis based on the CCTs in the eyes with corneal edema. The IOP measurements using 
Table 1. Comparison of central corneal thickness and intraocular pressure measurements in Group 1 and Group 2

\begin{tabular}{lcccc}
\hline & CCT $(\mu \mathrm{m})$ & GAT-IOP $(\mathrm{mmHg})$ & TAT-IOP $(\mathrm{mmHg})$ & DCT-IOP $(\mathrm{mmHg})$ \\
\hline Group 1 & $730.53 \pm 155.93$ & $17.33 \pm 7.87$ & $15.44 \pm 5.44$ & $15.85 \pm 6.25$ \\
Group 2 & $518.77 \pm 44.13$ & $13.06 \pm 3.70$ & $14.28 \pm 2.98$ & $14.28 \pm 3.05$ \\
P-value & $\mathrm{p}<0.001$ & $\mathrm{p}<0.001$ & $\mathrm{p}=0.067$ & $\mathrm{p}=0.028$ \\
\hline
\end{tabular}

Group 1: Postoperative edematous eyes, Group 2: Non-edematous fellow eyes

CCT: Central corneal thickness, GAT-IOP: Intraocular pressure by Goldmann applanation tonometer, TAT-IOP: Intraocular pressure by Tono-Pen, DCT-IOP: Intraocular pressure by Digital contour tonometer

GAT and TAT in the eyes with corneal edema were inversely proportional to the CCT $(\mathrm{p}=0.011$ and $\mathrm{p}=$ 0.033, respectively), but the relationship with DCT was not statistically significant $(p=0.44)$. These results may suggest that DCT measurements are less affected by corneal edema. The lack of a significant relationship between DCT and CCT in edematous corneas may suggest that DCT, as in normal corneas, can perform CCT-independent measurements. When the cornea is thicker, more force is required to compress a certain area of the tissue to be flattened, therefore the measured IOP values should be increased. However, if corneal edema is present, the water penetrates into the corneal layers and the corneal stroma swells like a sponge soaked in water, thus less force is required to straighten the cornea and the measured IOP values should be reduced. In a study performed in enucleated eyes with corneal edema (15); explained that the area of application with a fixed force is greater in eyes with corneal edema than eyes without corneal edema. The inverse correlation of TAT and GAT with CCT can be explained with the situation defined above. In the control group, there was only a significant correlation between the CCT and GAT IOP readings ( $\mathrm{p}<$ 0.001). This result shows that GAT is affected by CCT in normal corneas. It has already been shown in the literature that GAT is affected by CCT, and several correction tables were suggested (4-6). A limitation of the study was the lack of intraocular manometric measurements. Thus, we do not know precisely which method is the most appropriate and accurate.

There are several sources of error affecting the GAT IOP measurements (16). For example, significant increases in the CCT due to corneal edema can cause incorrect low GAT measurements. Simon et al. performed corneal hydration procedures in human cadaveric eyes, recorded the GAT IOP measurements, and determined that the mean IOP was significantly lower in the edematous eyes (17). The GAT IOP readings decreased by $9.2 \pm 0.7$ $\mathrm{mmHg}$ as the cornea was taken from minimum to maximum hydration. Conversely, in our study, the mean GAT IOP value in Group 1 was statistically significantly higher than that in Group $2(\mathrm{p}<0.001)$. This elevation may have been associated with cataract surgery. Lack of preoperative measurements is a limitation of the study.

DCT is an IOP measurement method that uses an optimized pressure-sensitive contact surface that minimizes corneal deformation. Several studies have shown that DCT readings are independent of the CCT and corneal edema (5, 18-20). However, some authors have determined that DCT is not entirely independent of corneal edema and the CCT (21-24). For example, Oh et al. examined 40 eyes of 20 healthy volunteer subjects, and they measured the CCT and IOP using DCT and GAT before and 2 hours after soft contact lens use (21). While there was an increase in the CCT, a decrease in the IOP measurements was also observed. The decrease in the IOP was statistically significant in the DCT measurements $(\mathrm{p}=$ $0.010)$, but not significant in the GAT measurements $(p=0.175)$. Moreover, Kniestedt et al. (18) measured the CCT and IOP using DCT, GAT, and pneumotonometry (PTG) in 10 freshly enucleated eyes with different hydrations. Two tubes placed in opposite directions in the anterior chamber were bound to a transducer, and the tube system was filled with a balanced salt solution. Due to the measurements, the GAT and PTG devices continuously showed lower IOP readings than the manometer. In the pressure range described by the bottle height of $10-50 \mathrm{~cm}$, the IOP readings measured using DCT were $0.50 \mathrm{mmHg} \quad[95 \%$ confidence interval $(\mathrm{CI})=0.40-0.60]$ and $0.36 \mathrm{mmHg}$ $(95 \%$ CI $=0.25-0.47)$ higher than the manometric measurements before and after dehydration, respectively. Only DCT showed no significant difference in the manometric reference measurements between the hydrated and dehydrated corneas. In our study, the mean IOP values in Group 1 were significantly higher when using GAT and DCT than they were in Group $2(\mathrm{p}<0.001$ and $\mathrm{p}=0.028$, respectively). The high GAT and DCT IOP values in Group 1 may have been associated with the cataract surgery. TAT tends to underestimate IOP compared to GAT and DCT readings in Group 1. TAT results differ especially in case of higher IOP values (25-27). 
Table 2. Comparison of compatibility of the three method pairs in both groups

\begin{tabular}{|c|c|c|c|c|c|c|c|c|c|}
\hline \multirow[t]{2}{*}{ Groups } & \multirow[t]{2}{*}{ Methods } & \multirow[t]{2}{*}{ Number } & \multirow{2}{*}{$\begin{array}{c}\text { Intraclass } \\
\text { correlation } \\
\text { coeffiecient }\end{array}$} & \multirow{2}{*}{$\begin{array}{l}\text { Mean of } \\
\text { difference } \\
(\mathrm{mmHg})\end{array}$} & \multirow{2}{*}{$\begin{array}{l}\text { Standard } \\
\text { deviation } \\
\text { (SD) }\end{array}$} & \multicolumn{2}{|c|}{$\begin{array}{l}\text { Limits of Agreement } \\
(\mathrm{mmHg})\end{array}$} & \multicolumn{2}{|c|}{$\begin{array}{c}\text { Bland-Altman } \\
\text { Analysis }\end{array}$} \\
\hline & & & & & & Mear & $96 * S D$ & $\mathrm{R}$ square & $P$ value \\
\hline \multirow[t]{3}{*}{ Group 1} & TAT-GAT & 64 & $\begin{array}{c}0.800(95 \% \mathrm{CI}= \\
0.656-0.881)\end{array}$ & -1.89 & 5.34 & -12.35 & 8.57 & 0.24 & $\mathrm{p}<0.001$ \\
\hline & TAT-DCT & 64 & $\begin{array}{c}0.809(95 \% \mathrm{CI}= \\
0.685-0.884)\end{array}$ & -0.41 & 4.71 & -9.64 & 8.82 & 0.04 & $\mathrm{p}=0.141$ \\
\hline & GAT-DCT & 64 & $\begin{array}{c}0.799(95 \% \mathrm{CI}= \\
668-878)\end{array}$ & 1.48 & 5.71 & -9.72 & 12.68 & 0.10 & $\mathrm{p}=0.013$ \\
\hline \multirow[t]{3}{*}{ Group 2} & TAT-GAT & 64 & $\begin{array}{c}0.894(95 \% \mathrm{CI}= \\
0.691-0.952)\end{array}$ & 1.22 & 1.78 & -2.26 & 4.70 & 0.17 & $\mathrm{p}=0.001$ \\
\hline & TAT-DCT & 64 & $\begin{array}{c}0.998(95 \% \mathrm{CI}= \\
0.997-0.999)\end{array}$ & 0 & 0.27 & -0.53 & 0.53 & 0.06 & $\mathrm{p}=0.053$ \\
\hline & GAT-DCT & 64 & $\begin{array}{c}0.895(95 \% \mathrm{CI}= \\
0.696-0.952)\end{array}$ & -1.22 & 1.80 & -4.74 & 2.30 & 0.14 & $\mathrm{p}=0.002$ \\
\hline
\end{tabular}

Group 1: Postoperative edematous eyes, Group 2: Non-edematous fellow eyes

GAT: Goldmann applanation tonometer, TAT: Tono-Pen applanation tonometer, DCT: Digital contour tonometer

This result might be due to high IOP measurements. In several studies comparing GAT and DCT in normal eyes, it was shown that DCT tends to measure higher IOP measurements than GAT (1, 28). Similarly, in our study, we found higher IOP measurements with DCT than GAT in normal eyes. However, we obtained lower IOP values with DCT than GAT in edematous eyes. This result might be due to the change in the biomechanical properties of the cornea in edematous eyes, as mentioned above.

The Tono-Pen is a portable IOP measurement method, and some previous studies have indicated that TAT is influenced by the CCT $(8,9,29,30)$. Bhan et al. (10) showed that the CCT has less of an effect on the IOP measurements when using TAT than when using GAT in normal corneas (an increase of $0.23 \mathrm{mmHg} / 10 \mu \mathrm{m}$ with the Goldmann tonometer and $0.10 \mathrm{mmHg} / 10 \mu \mathrm{m}$ with the Tono-Pen). Moreover, some previous studies have shown that TAT is affected by the CCT in edematous eyes (31, 32). For example, Neuburger et al. (32) clamped 8 donor corneas that were not appropriate for keratoplasty into an artificial anterior chamber. Stromal edema was present in all of the corneas. The intracameral pressure was adjusted to different values, and the CCT and IOP measurements using GAT, an I-Care tonometer, TAT, and an ocular response analyzer were obtained. The TAT and I-Care tonometer measurements were found to be the measurements most compatible with the manometric intracameral pressure values. In our study, the GAT and TAT IOP measurements of the edematous eyes were inversely proportional to the CCT $(p=0.011$ and $\mathrm{p}=0.033$, respectively), but the relationship with DCT was not statistically significant $(p=0.44)$. These results may suggest that corneal edema affected the
DCT measurements less than the GAT and TAT measurements. However, there is no gold standard measurement method in edematous corneas. Because we do not know the actual IOP value, it is not possible to claim that DCT is the most reliable and suitable method for IOP measurement.

There are conflicting results in studies evaluating the effect of age on IOP. Although there are studies with positive correlations between age and IOP (33-35), there are also studies with negative correlations (36, 37). In our study, the mean age of the patients was relatively high. That might have an effect on IOP measurements.

Our study did have some limitations. For example, GAT was the first method used for the IOP measurement and TAT was the second; therefore, the GAT measurements may have had an impact on the TAT measurements associated with corneal applanation. Another limitation of this study was the lack of endothelial cell count, peripheral corneal thickness, and preoperative CCT and IOP measurements. Moreover, this study included an elderly population, which could have confounded the results due to age-related changes. The influence of examiner bias might be more significant if GAT is carried out after TAT or DCT. GAT reading is more operator dependent, and thus, might be affected if the operator is aware of the TAT and DCT readings. Additionally, the intraobserver variability of the GAT IOP measurements could be evaluated in more detail. Future studies including patients with a specific age range, a larger number of patients, comparing more measurement methods including intraocular manometric measurements, and performing 
preoperative and postoperative measurements could help to clarify this issue.

In conclusion, we found the accordance between TAT and DCT measurements. However, this accordance does not mean that they are able to measure more accurate in edematous corneas. The lack of a significant relationship between DCT and CCT in edematous corneas may suggest that DCT, as in normal corneas, can perform CCT-independent measurements. However, there is no gold standard method for IOP measurement in edematous corneas. Therefore, an IOP assessment in patients with postoperative corneal edema is clinically challenging. Corneal biomechanical changes occur with corneal edema, thus there is still no gold standard method for a reliable and accurate IOP measurement. There is a need for further studies using advanced measurement methods compared with intraocular manometric measurements.

Disclosure of Interest: The authors declare that they have no competing interest.

\section{References}

1. Cook JA, Botello AP, Elders A, et al. Systematic review of the agreement of tonometers with Goldmann applanation tonometry. Ophthalmology 2012; 119: 1552-1557.

2. IOf S. Ophthalmic Instruments-Tonometers: ISO8612: 2001. Geneva, Switzerland: International Organization for Standardization. 2001.

3. Galgauskas S, Strupaite R, Strelkauskaite E, Asoklis R. Comparison of intraocular pressure measurements with different contact tonometers in young healthy persons. Int J Ophthalmol 2016; 9: 76-80.

4. Gunvant P, O'Leary DJ, Baskaran M, Broadway DC, Watkins RJ, Vijaya L. Evaluation of tonometric correction factors. J Glaucoma 2005; 14: 337-343.

5. Schneider E, Grehn F. Intraocular pressure measurement-comparison of dynamic contour tonometry and goldmann applanation tonometry. J Glaucoma 2006; 15: 2-6.

6. Kohlhaas M, Boehm AG, Spoerl E, Pursten A, Grein HJ, Pillunat LE. Effect of central corneal thickness, corneal curvature, and axial length on applanation tonometry. Arch Ophthalmol 2006; 124: 471-476.

7. Hessemer V, Rössler R, Jacobi KW. Tono-Pen, a new tonometer. International Ophthalmology 1989; 13: 51-56.

8. Tonnu PA, Ho T, Newson T, et al. The influence of central corneal thickness and age on intraocular pressure measured by pneumotonometry, non- contact tonometry, the Tono-Pen XL, and Goldmann applanation tonometry. $\mathrm{Br}$ J Ophthalmol 2005; 89: 851-854.

9. Dohadwala AA, Munger R, Damji KF. Positive correlation between Tono-Pen intraocular pressure and central corneal thickness. Ophthalmology 1998; 105: 1849-1854.

10. Bhan A, Browning AC, Shah S, Hamilton R, Dave D, Dua HS. Effect of corneal thickness on intraocular pressure measurements with the pneumotonometer, Goldmann applanation tonometer, and Tono-Pen. Investigative ophthalmology \& visual science 2002; 43: 1389 1392.

11. Kanngiesser HE, Kniestedt C, Robert YC. Dynamic contour tonometry: presentation of a new tonometer. J Glaucoma 2005; 14: 344-350.

12. Liu J, Roberts CJ. Influence of corneal biomechanical properties on intraocular pressure measurement: quantitative analysis. J Cataract Refract Surg 2005; 31: 146-155.

13. Hamilton KE, Pye DC, Kao L, Pham N, Tran AQ. The effect of corneal edema on dynamic contour and goldmann tonometry. Optom Vis Sci 2008; 85: 451-456.

14. Herr A, Remky A, Hirsch T, Rennings C, Plange $\mathrm{N}$. Tonometry in corneal edema after cataract surgery: dynamic contour tonometry versus Goldmann applanation tonometry. Clin Ophthalmol 2013; 7: 815-859.

15. Stelzer R. Relationship of the corneal applanations to intraocular pressure in enucleated human eyes (author's transl). Albrecht von Graefes Archiv fur klinische und experimentelle Ophthalmologie Albrecht von Graefe's archive for clinical and experimental ophthalmology 1973; 189: 33 .

16. Whitacre MM, Stein R. Sources of error with use of Goldmann-type tonometers. Surv Ophthalmol 1993; 38: 1-30.

17. Simon G, Small RH, Ren Q, Parel JM. Effect of corneal hydration on Goldmann applanation tonometry and corneal topography. Refract Corneal Surg 1993; 9: 110-117.

18. Kniestedt C, Nee M, Stamper RL. Accuracy of dynamic contour tonometry compared with applanation tonometry in human cadaver eyes of different hydration states. Graefes Arch Clin Exp Ophthalmol 2005; 243: 359-366.

19. Hsu SY, Sheu MM, Hsu AH, et al. Comparisons of intraocular pressure measurements: Goldmann applanation tonometry, noncontact tonometry, Tono-Pen tonometry, and dynamic contour tonometry. Eye (Lond) 2009; 23: 1582-1588.

20. Kotecha A, White ET, Shewry JM, Garway-Heath DF. The relative effects of corneal thickness and age on Goldmann applanation tonometry and dynamic contour tonometry. British Journal of Ophthalmology 2005; 89: 1572-1575. 
21. Oh JH, Yoo C, Kim YY, Kim HM, Song JS. The effect of contact lens-induced corneal edema on Goldmann applanation tonometry and dynamic contour tonometry. Graefes Arch Clin Exp Ophthalmol 2009; 247: 371-375.

22. Doyle A, Lachkar Y. Comparison of Dynamic Contour Tonometry With Goldman Applanation Tonometry Over a Wide Range of Central Corneal Thickness. Journal of Glaucoma 2005; 14: 288-292.

23. Francis BA, Hsieh A, Lai MY, et al. Effects of corneal thickness, corneal curvature, and intraocular pressure level on Goldmann applanation tonometry and dynamic contour tonometry. Ophthalmology 2007; 114: 20-26.

24. Milla E, Duch S, Buchacra O, Masuet C. Poor agreement between Goldmann and Pascal tonometry in eyes with extreme pachymetry. Eye (Lond) 2009; 23: 536-542.

25. Frenkel REP, Hong YJ, Shin DH. Comparison of the Tono-Pen to the Goldmann Applanation Tonometer. JAMA Ophthalmology 1988; 106: 750-753.

26. Horowitz GS, Byles J, Lee J, D'Este C. Comparison of the Tono-Pen and Goldmann tonometer for measuring intraocular pressure in patients with glaucoma. Clin Exp Ophthalmol 2004; 32: 584-589.

27. Doering CJ, Feldman E, Bdolah-Abram T, Merideth RE, Ofri R. Mathematical Discrepancies of the Tono-Pen Applanation Tonometer. Journal of Glaucoma 2017; 26: 30-36.

28. Kouchaki B, Hashemi H, Yekta A, khabazkhoob M. Comparison of current tonometry techniques in measurement of intraocular pressure. Journal of Current Ophthalmology 2017; 29: 92-97.

29. Sahin A, Basmak H, Yildirim N. The Influence of Central Corneal Thickness and Corneal Curvature on Intraocular Pressure Measured by Tono-Pen and Rebound Tonometer in Children. Journal of Glaucoma 2008; 17: 57-61.
30. Yildirim N, Sahin A, Basmak H, Bal C. Effect of central corneal thickness and radius of the corneal curvature on intraocular pressure measured with the Tono-Pen and noncontact tonometer in healthy schoolchildren. J Pediatr Ophthalmol Strabismus 2007; 44: 216-222.

31. Browning AC, Bhan A, Rotchford AP, Shah S, Dua HS. The effect of corneal thickness on intraocular pressure measurement in patients with corneal pathology. British Journal of Ophthalmology 2004; 88: 1395-1399.

32. Neuburger M, Maier P, Böhringer D, Reinhard T, F. Jordan J. The Impact of Corneal Edema on Intraocular Pressure Measurements Using Goldmann Applanation Tonometry, Tono-Pen XL, iCare, and ORA: An In Vitro Model. Journal of Glaucoma. 2013;22(7):584-90.

33. Cone FE, Steinhart MR, Oglesby EN, Kalesnykas G, Pease ME, Quigley HA. The effects of anesthesia, mouse strain and age on intraocular pressure and an improved murine model of experimental glaucoma. Experimental Eye Research 2012; 99: 27-35.

34. Rochtchina E, Mitchell P, Wang JJ. Relationship between age and intraocular pressure: the Blue Mountains Eye Study. Clinical \& Experimental Ophthalmology 2002; 30: 173-175.

35. Tonnu P-A, Ho T, Newson T, et al. The influence of central corneal thickness and age on intraocular pressure measured by pneumotonometry, noncontact tonometry, the Tono-Pen XL, and Goldmann applanation tonometry. British Journal of Ophthalmology 2005; 89: 851-854.

36. Lin H-Y, Hsu W-M, Chou P, et al. Intraocular Pressure Measured With a Noncontact Tonometer in an Elderly Chinese Population: The Shihpai Eye Study. JAMA Ophthalmology 2005; 123: 381-386.

37. Nomura H, Ando F, Niino N, Shimokata $H$, Miyake $\mathrm{Y}$. The relationship between age and intraocular pressure in a Japanese population: the influence of central corneal thickness. Current eye research 2002; 24: 81-85. 